\title{
SOJOURN TIMES FOR THE BROWNIAN MOTION
}

\author{
LAJOS TAKÁCS \\ Case Western Reserve University \\ Cleveland, $O H \quad U S A$
}

(Received September, 1997; Revised February, 1998)

In this paper explicit formulas are given for the distribution function, the density function and the moments of the sojourn time for the reflecting Brownian motion process.

Key words: Brownian Motion, Reflecting Brownian Motion, Sojourn Times, Distribution Functions, Moments.

AMS subject classifications: $60 \mathrm{~J} 15,60 \mathrm{~J} 55,60 \mathrm{~J} 65$.

\section{Introduction}

Let $\{\xi(t), t \geq 0\}$ be a standard Brownian motion process. We have $\mathbf{P}\{\xi(t) \leq x\}=$ $\Phi(x / \sqrt{t})$ for $t>0$ where

$$
\Phi(x)=\frac{1}{\sqrt{2 \pi}} \int_{-\infty}^{x} e^{-u^{2} / 2} d u
$$

is the normal distribution function. We also use the notation

$$
\varphi(x)=\frac{1}{\sqrt{2 \pi}} e^{-x^{2} / 2}
$$

for the normal density function.

Let us define

$$
\tau(\alpha)=\lim _{\varepsilon \rightarrow 0} \frac{1}{\varepsilon} \text { measure }\{t: \alpha \leq \xi(t)<\alpha+\varepsilon, 0 \leq t \leq 1\}
$$

for any real $\alpha$. The limit (3) exists with probability one and $\tau(\alpha)$ is a nonnegative random variable which is called the local time at level $\alpha$. We define also

$$
\omega(\alpha)=\int_{0}^{1} \delta(\xi(t)>\alpha) d t
$$

for $\alpha \geq 0$ where $\delta(S)$ denotes the indicator variable of any event $S$, that is, $\delta(S)=1$ if $S$ occurs and $\delta(S)=0$ if $S$ does not occur. The integral (4) exists with probability one and $\omega(\alpha)$ is a nonnegative random variable which is called the sojourn time of 
the process $\{\xi(t), t \geq 0\}$ spent in the set $(\alpha, \infty)$ in the time interval $(0,1)$. We also consider the reflecting Brownian motion process $\{|\xi(t)|, t \geq 0\}$ and define

$$
\omega^{*}(\alpha)=\int_{0}^{1} \delta(|\xi(t)|>\alpha) d t
$$

for $\alpha \geq 0$ as the sojourn time of the process $\{|\xi(t)|, t \geq 0\}$ spent in the set $(\alpha, \infty)$ in the time interval $(0,1)$.

Our main object is to determine the distribution and the moments of $\omega^{*}(\alpha)$ for $\alpha>0$. In principle, we can apply the method of M. Kac [6] to find the distribution of $\omega^{*}(\alpha)$. His method requires the inversion of a double Laplace transform which can be obtained by solving a certain Sturm-Liouville differential equation. Our approach is combinatorial and we shall find explicit formulas for the distribution function and the moments of $\omega^{*}(\alpha)$.

Let us define

and

$$
\begin{aligned}
& \mathbf{E}\left\{[\tau(\alpha)]^{r}\right\}=m_{r}(\alpha), \\
& \mathbf{E}\left\{[\omega(\alpha)]^{r}\right\}=M_{r}(\alpha)
\end{aligned}
$$

$$
\mathbf{E}\left\{\left[\omega^{*}(\alpha)\right]^{r}\right\}=M_{r}^{*}(\alpha)
$$

for $r=1,2, \ldots$ and $\alpha \geq 0$. We shall prove the following surprisingly simple formulas for the moments (7) and (8):

and

$$
M_{r}(\alpha)=m_{2 r}(\alpha) /\left(2^{r} r !\right)
$$

$$
M_{r}^{*}(\alpha)=\frac{(r-1) !}{2^{r-1}} \sum_{k=1}^{r} \frac{m_{2 r}((2 k-1) \alpha)}{(r-k) !(r+k-1) !}
$$

if $r=1,2, \ldots$ and $\alpha>0$. Equations (9) and (10) make it possible to determine the distribution function $\mathbf{P}\left\{\omega^{*}(\alpha) \leq x\right\}=G_{\alpha}(x)$ explicitly. We shall prove that

$$
\begin{aligned}
& G_{\alpha}(x) \\
& \quad=2 F_{\alpha}(x)-1+2 \sum_{k=2}^{\infty} \sum_{j=2}^{k} \frac{(-1)^{j} j !}{(k+j-1) !}\left(\begin{array}{c}
k-2 \\
j-2
\end{array}\right) \frac{d^{k-1} x^{k-1}\left[1-F_{(2 j-1) \alpha}(x)\right]}{d x^{k-1}}
\end{aligned}
$$

if $0 \leq x<1$ and $\alpha>0$, and $G_{\alpha}(1)=1$. In (11), $F_{\alpha}(x)=\mathbf{P}\{\omega(\alpha) \leq x\}$. We have

for $0<x \leq 1$, and $\alpha \geq 0$, and

$$
F_{\alpha}(x)=1-\frac{1}{\pi} \int_{0}^{1-x} \frac{e^{-\alpha^{2} /(2 u)}}{\sqrt{u(1-u)}} d u
$$

$$
F_{\alpha}(0)=2 \Phi(\alpha)-1
$$

for $\alpha \geq 0$. The distribution function $F_{\alpha}(x)$ was found by P. Lévy [9] p. 326 in 1939 . If, in particular, $x=0$ in (11), we obtain that 
$G_{\alpha}(0)$

$$
=1+4 \sum_{k=1}^{\infty}(-1)^{k}[1-\Phi((2 k-1) \alpha)]=\frac{4}{\pi} \sum_{j=1}^{\infty} \frac{(-1)^{j-1}}{2 j-1} e^{-(2 j-1)^{2} \pi^{2} /\left(8 \alpha^{2}\right)}
$$

for $\alpha>0$.

We note that

$$
\mathbf{P}\{\tau(\alpha) \leq x\}=2 \Phi(\alpha+x)-1
$$

if $x \geq 0$ and $\alpha \geq 0$, and

$$
m_{r}(\alpha)=2 r \int_{0}^{\infty} x^{r-1}[1-\Phi(\alpha+x)] d x
$$

if $\alpha \geq 0$ and $r \geq 1$ where $\Phi(x)$ is defined by (1). Explicitly,

$$
m_{r}(\alpha)=2(-1)^{r}\left\{a_{r}(\alpha)[1-\Phi(\alpha)]-b_{r}(\alpha) \varphi(\alpha)\right\}
$$

for $r=1,2, \ldots$ where

and

$$
a_{r}(\alpha)=r ! \sum_{j=0}^{[r / 2]} \frac{\alpha^{r-2 j}}{2^{j} j !(r-2 j) !}
$$

$$
b_{r}(\alpha)=\sum_{j=0}^{[(r-1) / 2]}\left(\begin{array}{c}
r-1-j \\
j
\end{array}\right) \frac{j ! \alpha^{r-1-2 j}}{2^{j}} \sum_{\nu=0}^{j}\left(\begin{array}{l}
r \\
\nu
\end{array}\right)
$$

for $r \geq 1$. See L. Takács [13].

Our approach is based on a symmetric random walk $\left\{\zeta_{r}, r \geq 0\right\}$ where $\zeta_{r}=\xi_{1}+$ $\xi_{2}+\ldots+\xi_{r}$ for $r \geq 1, \zeta_{0}=0$, and $\left\{\xi_{r}, r \geq 1\right\}$ is a sequence of independent and identically distributed random variables for which

$$
\mathbf{P}\left\{\xi_{r}=1\right\}=\mathbf{P}\left\{\xi_{r}=-1\right\}=1 / 2 .
$$

Let us define $\tau_{n}(a)$ as the number of subscripts $r=0,1, \ldots, n$ for which $\zeta_{r}=a$ where $a=0,1,2, \ldots$. Furthermore, define $\omega_{n}(a)$ as the number of subscripts $r=0$, $1, \ldots, n$ for which $\zeta_{r} \geq a$ where $a=0,1,2, \ldots$, and $\omega_{n}^{*}(a)$ as the number of subscripts $r=1,2, \ldots, n$ for which $\left|\zeta_{r}\right| \geq a$ where $a=1,2, \ldots$.

By the results of M.D. Donsker [2], if $n \rightarrow \infty$, the process $\left\{\zeta_{[n t]} / \sqrt{n}, 0 \leq t \leq 1\right\}$ converges weakly to the Brownian motion $\{\xi(t), 0 \leq t \leq 1\}$. See also I.I. Gikhman and A.V. Skorokhod [4] pp. 490-495.

In 1965, F.B. Knight [7] proved that

$$
\lim _{n \rightarrow \infty} \mathbf{P}\left\{\frac{\tau_{n}([\alpha \sqrt{n}])}{\sqrt{n}} \leq x\right\}=\mathbf{P}\{\tau(\alpha) \leq x\}
$$

for $\alpha \geq 0$ and $x>0$. Since the integrals (4) and (5) are continuous functionals of the process $\{\xi(t), 0 \leq t \leq 1\}$, we can conclude that

and

$$
\lim _{n \rightarrow \infty} \mathbf{P}\left\{\omega_{n}([\alpha \sqrt{n}]) \leq n x\right\}=\mathbf{P}\{\omega(\alpha) \leq x\}
$$




$$
\lim _{n \rightarrow \infty} \mathbf{P}\left\{\omega_{n}^{*}([\alpha \sqrt{n})] \leq n x\right\}=\mathbf{P}\left\{\omega^{*}(\alpha) \leq x\right\}
$$

for $\alpha>0$ and $x \geq 0$.

We shall determine the distributions and the moments of the random variables $\tau_{n}(a), \omega_{n}(a)$ and $\omega_{n}^{*}(a)$, and their asymptotic behavior in the case where $a=[\alpha \sqrt{n}]$, $\alpha>0$, and $n \rightarrow \infty$. We shall prove that

$$
\lim _{n \rightarrow \infty} \mathbf{E}\left\{\left(\frac{\tau_{n}([\alpha \sqrt{n}])}{\sqrt{n}}\right)^{r}\right\}=m_{r}(\alpha)
$$

for $r \geq 1$ and $\alpha \geq 0$ where $m_{r}(\alpha)$ is given by (16). Furthermore, we shall determine (7) and (8) by calculating the following limits

$$
\lim _{n \rightarrow \infty} \mathbf{E}\left\{\left(\frac{\omega_{n}([\alpha \sqrt{n}])}{n}\right)^{r}\right\}=M_{r}(\alpha)
$$

and

$$
\lim _{n \rightarrow \infty} \mathbf{E}\left\{\left(\frac{\omega_{n}^{*}([\alpha \sqrt{n}])}{n}\right)^{r}\right\}=M_{r}^{*}(\alpha)
$$

for $r \geq 1$ and $\alpha>0$. The moments $M_{r}(\alpha),(r \geq 1)$, and $M_{r}^{*}(\alpha),(r \geq 1)$, uniquely determine the distribution functions $\mathbf{P}\{\omega(\alpha) \leq x\}$ and $\mathbf{P}\left\{\omega^{*}(\alpha) \leq x\right\}$.

\section{The Random Walk $\left\{\zeta_{r}, r \geq 0\right\}$}

Let us recall some results for $\left\{\zeta_{r}, r \geq 0\right\}$ which we need in this paper. See L. Takács [12]. We have

$$
\mathbf{P}\left\{\zeta_{n}=2 j-n\right\}=\left(\begin{array}{c}
n \\
j
\end{array}\right) \frac{1}{2^{n}}
$$

for $j=0,1, \ldots, n$, and by the central limit theorem

where $\Phi(x)$ is defined by (1).

$$
\lim _{n \rightarrow \infty} \mathbf{P}\left\{\frac{\zeta_{n}}{\sqrt{n}} \leq x\right\}=\Phi(x)
$$

Let us define $\rho(a)$ as the first passage time through $a(a=0, \pm 1, \pm 2, \ldots)$, that is,

We have

$$
\rho(a)=\inf \left\{r: \zeta_{r}=a \text { and } r \geq 0\right\}
$$

$$
\mathbf{P}\{\rho(a)=a+2 j\}=\frac{a}{a+2 j}\left(\begin{array}{c}
a+2 j \\
j
\end{array}\right) \frac{1}{2^{a+2 j}}
$$

for $a \geq 1$ and $j \geq 0$. If $1 \leq a \leq n$, then

By (30),

$$
\mathbf{P}\{\rho(a) \leq n\}=\mathbf{P}\left\{\zeta_{n} \geq a\right\}+\mathbf{P}\left\{\zeta_{n}>a\right\} .
$$

$$
\sum_{n=0}^{\infty} \mathbf{P}\{\rho(a)=n\} w^{n}=[\gamma(w)]^{a}
$$

for $a \geq 1$ and $|w| \leq 1$ where $\gamma(0)=0$ and

$$
\gamma(w)=\left(1-\sqrt{1-w^{2}}\right) / w
$$


for $0<|w| \leq 1$. The identity

$$
\sum_{j=0}^{n} \mathbf{P}\{\rho(a)=j\} \mathbf{P}\{\rho(b)=n-j\}=\mathbf{P}\{\rho(a+b)=n\}
$$

is valid for any $a \geq 1, b \geq 1$ and $n \geq 1$.

We note that

$$
\mathbf{P}\{\rho(1)=2 n+1\}=C_{n} / 2^{2 n+1}
$$

for $n=0,1,2, \ldots$ where

is the $n$th Catalan number.

$$
C_{n}=\left(\begin{array}{c}
2 n \\
n
\end{array}\right) \frac{1}{n+1}
$$

Let us define

$$
P(n, \nu)=\sum_{\substack{\alpha_{1}+\alpha_{2}+\ldots+\alpha_{n}=\nu \\ \alpha_{1}+2 \alpha_{2}+\ldots+n \alpha_{n}=n}} \frac{\nu !}{\alpha_{1} ! \alpha_{2} ! \ldots \alpha_{n} !} C_{0}^{\alpha_{1}} C_{1}^{\alpha_{2}} \ldots C_{n-1}^{\alpha_{n}}
$$

for $1 \leq \nu \leq n$. To evaluate (37) let us express each Catalan number in (37) by (35). By the repeated applications of (34) we obtain that

$$
P(n, \nu)=2^{2 n-\nu} \mathbf{P}\{\rho(\nu)=2 n-\nu\}=\left(\begin{array}{c}
2 n-\nu \\
n
\end{array}\right) \frac{\nu}{2 n-\nu}
$$

By (31) we obtain that

$$
\begin{gathered}
\sum_{s=j}^{r} \mathbf{P}\{\rho(2 j)=2 s\}=\mathbf{P}\{\rho(2 j) \leq 2 r+1\} \\
=2 \mathbf{P}\left\{\zeta_{2 r+1} \geq 2 j+1\right\}=\sum_{s=j}^{r}\left(\begin{array}{c}
2 r+1 \\
r-s
\end{array}\right) \frac{1}{2^{2 r}}
\end{gathered}
$$

for $j=0,1, \ldots, r$.

If $a \geq 1$ and $b \geq 1$, let us define $\Theta(a, b)$ as the smallest $r=0,1, \ldots$ for which either $\zeta_{r}=a$ or $\zeta_{r}=-b$. We can interpret $\Theta(a, b)$ as the duration of games in the classical ruin problem. See L. Takács [11]. By the results of P.S. Laplace [8], p. 228 we have

$$
\mathbf{E}\left\{w^{\Theta(a, b)}\right\}=\frac{[\gamma(w)]^{a}+[\gamma(w)]^{b}}{1+[\gamma(w)]^{a+b}}
$$

if $|w| \leq 1$ where $\gamma(w)$ is defined by (33). See also I. Todhunter [15], p. 169 .

\section{Sojourn Times}

Let us consider now a stochastic process $\{(t), t \geq 0\}$ with state space $A \cup B$ where $A$. and $B$ are disjoint Borel sets. Let $\mathbf{P}\{\zeta(0) \in A\}=1$ and denote by $\alpha_{1}, \beta_{1}, \alpha_{2}, \beta_{2}, \ldots$ the lengths of the successive intervals spent in states $A$ and $B$ respectively in the interval $(0, \infty)$. We suppose that $\left\{\alpha_{i}\right\}$ and $\left\{\beta_{i}\right\}$ are discrete random variables which take on positive integers only. Define $\gamma_{n}=\alpha_{1}+\alpha_{2}+\ldots+\alpha_{n}$ for $n \geq 1$ and $\gamma_{0}=0$. Furthermore, let $\delta_{n}=\beta_{1}+\beta_{2}+\ldots+\beta_{n}$ for $n \geq 1$ and $\delta_{0}=0$.

Denote by $\beta(n+1)$ the total time spent in state $B$ in the interval $(0, n+1)$. If the 
two sequences $\left\{\alpha_{i}\right\}$ and $\left\{\beta_{i}\right\}$ are independent, then we have

$$
\mathbf{P}\{\beta(n+1) \leq k\}=\sum_{r \geq 0} \mathbf{P}\left\{\delta_{r} \leq k\right\}\left[\mathbf{P}\left\{\gamma_{r} \leq n-k\right\}-\mathbf{P}\left\{\gamma_{r+1} \leq n-k\right\}\right]
$$

for $0 \leq k \leq n$.

Proof of (41): Denote by $\alpha(t)$ the total time spent in state $A$ in the time interval $(0, t)$ and by $\beta(t)$ the total time spent in state $B$ in the time interval $(0, t)$. If $0 \leq$ $k \leq n$, denote by $\tau=\tau(n-k)$ the smallest $u \in[0, \infty)$ for which $\alpha(u)=n-k+1$. Then we have $\{\beta(n+1) \leq k\} \equiv\{\beta(\tau) \leq k\}$. This follows from the following identities

$$
\begin{gathered}
\{\beta(n+1) \leq k\} \equiv\{\alpha(\tau) \leq \alpha(n+1)\} \equiv\{\tau \leq n+1\} \\
\equiv\{\alpha(\tau)+\beta(\tau) \leq n+1\} \equiv\{\beta(\tau) \leq k\}
\end{gathered}
$$

Since $\beta(\tau)=\delta_{r}(r=0,1,2, \ldots)$ if $\gamma_{r}<n+1-k \leq \gamma_{r+1}$, it follows from (42) that

$$
\mathbf{P}\{\beta(n+1) \leq k\}=\sum_{r \geq 0} \mathbf{P}\left\{\delta_{r} \leq k \text { and } \gamma_{r} \leq n-k<\gamma_{r+1}\right\}
$$

for $0 \leq k \leq n$. This proves (41).

By forming generating functions, we obtain from (41) that

$$
\begin{gathered}
(1-w)(1-z w) \sum_{n=0}^{\infty} \mathbf{E}\left\{z^{\beta(n+1)}\right\} w^{n} \\
=(1-w) z+(1-z) \sum_{r=0}^{\infty} \mathbf{E}\left\{(z w)^{\delta} r\right\}\left[\mathbf{E}\left\{w^{\gamma} r\right\}-\mathbf{E}\left\{w^{\gamma r+1}\right\}\right]
\end{gathered}
$$

if $|w|<1$ and $|z w|<1$.

Now we consider the case where $\left\{\alpha_{i}\right\}$ and $\left\{\beta_{i}\right\}$ are independent sequences of independent random variables such that $\alpha_{2}, \alpha_{3}, \ldots$ are identically distributed, but $\alpha_{1}$ may have a different distribution, and $\beta_{1}, \beta_{2}, \ldots$ are identically distributed. Let us write $\mathbf{E}\left\{z^{\alpha_{1}}\right\}=a_{0}(z), \mathbf{E}\left\{z^{\alpha_{i}}\right\}=a(z)$ for $i=2,3, \ldots$ and $\mathbf{E}\left\{z^{\beta_{i}}\right\}=b(z)$ for $i=1,2, \ldots$ In this case by (44) we have

$$
(1-w)(1-z w) \sum_{n=0}^{\infty} \mathbf{E}\left\{z^{\beta(n+1)}\right\} w^{n}=1-z w-(1-z) \frac{[1-b(z w)] a_{0}(w)}{1-a(w) b(z w)}
$$

if $|w|<1$ and $|z w|<1$.

\section{On a Formula of Faà di Bruno}

The $n$th derivative of the compound function $f=f(y)$ where $y=y(z)$ is given by Faà di Bruno's formula

$$
\frac{d^{n} f}{d z^{n}}=\sum_{\nu=1}^{n} \frac{d^{\nu} f}{d y^{\nu}} Y_{n, \nu}(y)
$$

where

$Y_{n, \nu}(y)$ 


$$
=\sum_{\substack{\alpha_{1}+\alpha_{2}+\ldots+\alpha_{n}=\nu \\ \alpha_{1}+2 \alpha_{2}+\ldots+n \alpha_{n}=n}} \frac{n !}{\alpha_{1} ! \alpha_{2} ! \ldots \alpha_{n} !}\left(\frac{y^{(1)}(z)}{1 !}\right)^{\alpha_{1}}\left(\frac{y^{(2)}(z)}{2 !}\right)^{\alpha_{2}} \ldots\left(\frac{y^{(n)}(z)}{n !}\right)^{\alpha_{n}} .
$$

See Faà di Bruno [3] and Ch. Jordan [5], p. 34.

In this paper we need to calculate the $r$ th derivative of a function of the form $f(\gamma(z w))$ where $\gamma(w)$ is given by (33) for $|w| \leq 1$. In what follows we use the abbreviation $\gamma=\gamma(w)$ for a fixed $w$. Since

$$
w \gamma^{2}-2 \gamma+w=0
$$

for $|w| \leq 1$, we can easily see that

$$
\gamma^{(i)}(w)=\frac{i !\left(1+\gamma^{2}\right)^{i+1}}{\left(1-\gamma^{2}\right)^{2 i-1}} g_{i}(\gamma)
$$

for $i=1,2, \ldots$ and $|w|<1$ where $g_{i}(x)$ is a polynomial of degree $3(i-1)$ in $x$. In particular, $2 g_{1}(x)=1,4 g_{2}(x)=3 x-x^{3}$ and $8 g_{3}(x)=1+11 x^{2}-5 x^{4}+x^{6}$. For the determination of $g_{i}(x), i \geq 1$, we have the recurrence formula

$$
(i+1) g_{i+1}(x)=\left[3 i x+(i-2) x^{3}\right] g_{i}(x)-\frac{1}{2}\left(x^{4}-1\right) g_{i}^{\prime}(x) .
$$

If we apply (46) to the function $f(\gamma(z w))$, we obtain that

$$
\left(\frac{d^{n} f(\gamma(z w))}{d z^{n}}\right)_{z=1}=\frac{2^{n} \gamma^{n}}{\left(1-\gamma^{2}\right)^{2 n}} \sum_{\nu=1}^{n} f^{(\nu)}(\gamma)\left(1-\gamma^{4}\right)^{\nu} Q_{n, \nu}(\gamma)
$$

where

$$
Q_{n, \nu}(\gamma)=\sum_{\substack{\alpha_{1}+\alpha_{2}+\ldots+\alpha_{n}=\nu \\ \alpha_{1}+2 \alpha_{2}+\ldots+n \alpha_{n}=n}} \frac{n !}{\alpha_{1} ! \alpha_{2} ! \ldots \alpha_{n} !}\left[g_{1}(\gamma)\right]^{\alpha_{1}}\left[g_{2}(\gamma)\right]^{\alpha_{2} \ldots\left[g_{n}(\gamma)\right]^{\alpha}}
$$

for $1 \leq \nu \leq n$. Clearly, $Q_{n, \nu}(\gamma)$ is a polynomial of degree $3(n-\nu)$ in $\gamma$.

By $(50)$ we obtain that $g_{i}(1)=C_{i-1} / 2$ for $i=1,2, \ldots$ where $C_{i-1}$ is a Catalan number defined by (36). By (38) we have

$$
Q_{n, \nu}(1)=\frac{n ! P(n, \nu)}{2^{\nu} \nu !}=\frac{\nu(2 n-1-\nu) !}{2^{\nu} \nu !(n-\nu) !}
$$

if $1 \leq \nu \leq n$. We have also

if $1 \leq i \leq n$.

$$
\sum_{\nu=i}^{n}\left(\begin{array}{c}
\nu-1 \\
i-1
\end{array}\right) P(n, \nu)=\frac{i}{n}\left(\begin{array}{c}
2 n \\
n-i
\end{array}\right)
$$

We shall use the definition and some properties of the Kummer hypergeometric function

$$
M(a, b, z)={ }_{1} F_{1}(a ; b ; z)=\sum_{n=0}^{\infty} \frac{(a)_{n}}{(b)_{n}} \frac{z^{n}}{n !}
$$

where $(a)_{0}=1$ and $(a)_{n}=a(a+1) \ldots(a+n-1)$ for $n \geq 1$. We have

$$
M(a, b,-z)=e^{-z} M(b-a, b, z)
$$


and

$$
\int_{0}^{\infty} t^{s-1} M(a, b,-t) d t=\frac{\Gamma(b) \Gamma(s) \Gamma(a-s)}{\Gamma(a) \Gamma(b-s)}
$$

if $0<R e(s)<R e(a)$. See L.J. Slater [10] and M. Abramowitz and I.A. Stegun [1].

\section{The Local Time $\tau_{n}(a)$}

We defined $\tau_{n}(a)$ as the number of subscripts $r=0,1,2, \ldots, n$ for which $\zeta_{r}=a$ where $a \geq 0$. If $a \geq 1$, then

$$
\mathbf{P}\left\{\tau_{n}(a)=0\right\}=\mathbf{P}\{\rho(a)>n\},
$$

and if $a \geq 1$ and $k \geq 1$, then

$$
\mathbf{P}\left\{\tau_{n}(a) \geq k\right\}=\mathbf{P}\{\rho(a+k-1) \leq n+1-k\} .
$$

The distribution of $\rho(a)$ is given by $(30)$.

Equation (58) is trivially true. To prove (59) let us denote by $\theta_{1}, \theta_{1}+\theta_{2}, \ldots, \theta_{1}+$ $\ldots+\theta_{i}, \ldots$ the successive values of $r=1,2, \ldots$ for which $\zeta_{r}=a$. The random variables $\theta_{i},(i \geq 1)$, are independent, $\theta_{1}$ has the same distribution as $\rho(a)$ and $\theta_{i},(i \geq 2)$, has the same distribution as $\rho(1)+1$. Since

$$
\mathbf{P}\left\{\tau_{n}(a) \geq k\right\}=\mathbf{P}\left\{\theta_{1}+\theta_{2}+\ldots+\theta_{k} \leq n\right\}
$$

we obtain (59) by (34).

We note that

if $1 \leq k \leq n$.

$$
\mathbf{P}\left\{\tau_{n}(0)>k\right\}=\mathbf{P}\{\rho(k) \leq n-k\}
$$

If in (59) we put $a=[\alpha \sqrt{n}]$ where $\alpha>0$ and $k=[x \sqrt{n}]$ where $x \geq 0$ and let $n \rightarrow \infty$, then by (28) and (31) we obtain that

$$
\lim _{n \rightarrow \infty} \mathbf{P}\left\{\frac{\tau_{n}([\alpha \sqrt{n}])}{\sqrt{n}} \leq x\right\}=2 \Phi(\alpha+x)-1
$$

for $x \geq 0$ and $\alpha>0$. This proves (21).

By (32) and (59) we can prove that

$$
\Psi_{r}(a)=(1-w) \sum_{n=0}^{\infty} \mathbf{E}\left\{\left(\begin{array}{c}
\tau_{n}(a) \\
r
\end{array}\right)\right\} w^{n}=\frac{2^{r-1} \gamma^{a+2 r-2}\left(1+\gamma^{2}\right)}{\left(1-\gamma^{2}\right)^{r}}
$$

if $|w|<1, r \geq 1$ and $a \geq 1$. In (63) we used the abbreviation $\gamma=\gamma(w)$, where $\gamma(w)$ is defined by (33), and we took into consideration that $w=2 \gamma /\left(1+\gamma^{2}\right)$.

We observe that if $n+a$ is odd, then $\tau_{n}(a)$ has the same distribution as $\tau_{n-1}(a)$. If $n+a$ is even, then by expanding (63) into Taylor series at $w=0$, we obtain that

$$
\mathbf{E}\left\{\left(\begin{array}{c}
\tau_{n}(a) \\
r
\end{array}\right)\right\}=\sum_{j=0}^{a+r-1}(-1)^{j}\left(\begin{array}{c}
a+r-1 \\
j
\end{array}\right)\left(\begin{array}{c}
(n+a+r-j) / 2 \\
(n+a) / 2
\end{array}\right)
$$


for $a \geq 1, n \geq 1$ and $r \geq 1$.

We can prove that

$$
\mathbf{E}\left\{\left(\begin{array}{c}
\tau_{n}(a) \\
r
\end{array}\right)\right\}=2^{r+1} \mathbf{E}\left\{\left(\begin{array}{c}
{\left[\left(\zeta_{n+1}-a\right) / 2\right]^{+}} \\
r
\end{array}\right)\right\}
$$

if $n+a$ is odd, and

if $n+a$ is even.

$$
\mathbf{E}\left\{\left(\begin{array}{c}
\tau_{n}(a) \\
r
\end{array}\right)\right\}=2^{r+1} \mathbf{E}\left\{\left(\begin{array}{c}
{\left[\left(\zeta_{n+2}-a\right) / 2\right]^{+}} \\
r
\end{array}\right)\right\}
$$

Theorem 1: If $a=[\alpha \sqrt{n}]$ where $\alpha>0$, then

$$
\lim _{n \rightarrow \infty} \mathbf{E}\left\{\left(\frac{\tau_{n}(a)}{\sqrt{n}}\right)^{r}\right\}=m_{r}(\alpha)
$$

for $r \geq 1$ where $m_{r}(\alpha)$ is given by (16).

Proof: If $a=[\alpha \sqrt{n}]$ where $\alpha>0$ and $n \rightarrow \infty$, then by (65) and (66) we obtain that

$$
\mathbf{E}\left\{\left[\tau_{n}(a)\right]^{r}\right\} \sim 2 \mathbf{E}\left\{\left(\left[\zeta_{n}-a\right]^{+}\right)^{r}\right\}
$$

for $r=1,2, \ldots$. Accordingly,

$$
\lim _{n \rightarrow \infty} \mathbf{E}\left\{\left(\frac{\tau_{n}([\alpha \sqrt{n}])}{\sqrt{n}}\right)^{r}\right\}=\lim _{n \rightarrow \infty} \mathbf{E}\left\{\left(\left[\frac{\zeta_{n}}{\sqrt{n}}-\alpha\right]^{+}\right)^{r}\right\}=2 \mathbf{E}\left\{\left([\xi-a]^{+}\right)^{r}\right\}
$$

for $r=1,2, \ldots$ where $\mathbf{P}\{\xi \leq x\}=\Phi(x)$ and $\Phi(x)$ is defined by (1). Since

$$
2 \mathbf{E}\left\{\left([\xi-a]^{+}\right)^{r}\right\}=m_{r}(\alpha)
$$

for $\alpha>0$ and $r \geq 1$, where $m_{r}(\alpha)$ is given by (16), (69) implies (67).

The limit theorem (67) proves (24). We note that if in (67) $a=a_{n}$ where $\lim _{n \rightarrow \infty} a_{n} / \sqrt{n}=\alpha>0$, then the right-hand side of (67) remains unchanged.

Finally, we note that if $n+a$ is odd then by (65) we can write that

and

$$
\mathbf{E}\left\{\tau_{n}(a)\right\}=(n+a+1) \mathbf{P}\left\{\zeta_{n}=a+1\right\}-2 a \mathbf{P}\left\{\zeta_{n} \geq a+1\right\}
$$

$\mathbf{E}\left\{\left[\tau_{n}(a)\right]^{2}\right\}$

$$
=2\left(n+a^{2}+a+1\right) \mathbf{P}\left\{\zeta_{n} \geq a+1\right\}-(n+a+1)(a+2) \mathbf{P}\left\{\zeta_{n}=a+1\right\} .
$$

Similar expressions can be derived for $\mathbf{E}\left\{\left[\tau_{n}(a)\right]^{r}\right\}$ if $r \geq 2$.

\section{The Sojourn Time $\omega_{n}(a)$}

We defined $\omega_{n}(a)$ as the number of subscripts $r=0,1,2, \ldots, n$ for which $\zeta_{r} \geq a$ where 
$a=0,1,2, \ldots$ If $1 \leq a \leq n$, then evidently

$$
\mathbf{P}\left\{\omega_{n}(a)=0\right\}=\mathbf{P}\{\rho(a)>n\}
$$

and the distribution of $\rho(a)$ is given by (30). If $1 \leq j \leq n+1-a$, then we can write that

$$
\mathbf{P}\left\{\omega_{n}(a)=j\right\}=\frac{1}{2} \mathbf{P}\{\rho(1) \geq j\}[\mathbf{P}\{\rho(a)>n-j\}-\mathbf{P}\{\rho(a-1)>n-j\}] .
$$

See Theorem 2 in L. Takács [14]. By (74) we can prove that

$$
\mathbf{P}\left\{\omega_{n}(a)=j\right\}=\left(\begin{array}{c}
j-1 \\
{[(j-1) / 2]}
\end{array}\right)\left(\begin{array}{c}
n-j \\
{[(n+1-a-j) / 2]}
\end{array}\right) \frac{1}{2^{n}}
$$

if $1 \leq j \leq n+1-a$. Since $\omega_{n}(0)$ has the same distribution as $n+1-\omega_{n}(1)$, we have

$$
\mathbf{P}\left\{\omega_{n}(0)=j\right\}= \begin{cases}\mathbf{P}\left\{\omega_{n}(1)=j\right\} & \text { if } 1 \leq j \leq n \\ \mathbf{P}\left\{\omega_{n}(1)=0\right\} & \text { if } j=n+1\end{cases}
$$

By using (74), it is easy to prove that (22) holds and that $\mathbf{P}\{\omega(\alpha) \leq x\}$ is given explicitly by (12) and (13).

Our next aim is to determine the binomial moments of $\omega_{n}(a)$. We shall show that the $r$ th binomial moment of $\omega_{n}(a)$ can be expressed as a linear combination of the $2 r$ th binomial moments of $\tau_{n}(a-3 r+k)$ for $k=1,2, \ldots, 3 r$.

By (74) we obtain that

$$
(1-w) \sum_{n=0}^{\infty} \mathbf{E}\left\{z^{\omega_{n}(a)}\right\} w^{n}=1-[\gamma(w)]^{a}+\frac{[1-\gamma(w)][\gamma(w)]^{a-1}[1-\gamma(z w)] z w}{2(1-z w)}
$$

if $|w|<1,|z w|<1$ and $a \geq 1$, where $\gamma(w)$ is defined by (33). If we form the $r$ th derivative of (77) with respect to $z$ at $z=1$, we get

where

$$
\Phi_{r}(a)=(1-w) \sum_{n=0}^{\infty} \mathbf{E}\left\{\left(\begin{array}{c}
\omega_{n}(a) \\
r
\end{array}\right)\right\} w^{n}=\frac{2^{r-1} \gamma^{a+r-1}\left(1+\gamma^{2}\right)}{\left(1-\gamma^{2}\right)^{2 r}} L_{r}(\gamma)
$$

$$
L_{r}(x)=(1+x)^{2 r}+(1-x)^{2}(1+x) g_{r}(x)-\left(1+x^{2}\right) \sum_{j=0}^{r}(1+x)^{2 r-2 j+1} g_{j}(x)
$$

is a polynomial of degree $<3 r$ in $x$. In (78) we used the abbreviation $\gamma=\gamma(w)$, and in (79), $g_{j}(x)$ is defined by (49). If we use the abbreviation $\Psi_{r}(a)$ for (63), suppressing $w$, then (78) can be expressed in the following way:

$$
2^{r} \Phi_{r}(a)=\Psi_{2 r}(a-3 r+1) L_{r}(\gamma)
$$

Since $\Psi_{r}(a) \gamma=\Psi_{r}(a+1)$ for any $r=1,2, \ldots$ and $a=1,2, \ldots$, the right-hand side of (80) can be expressed as a linear combination of $\Psi_{2 r}(a-3 r+k)$ for $k=1,2, \ldots, 3 r$. 
In particular, we have

and

$$
2 \Phi_{1}(a)=\Psi_{2}(a-2)+\Psi_{2}(a-1)
$$

Hence

and

$$
8 \Phi_{2}(a)=\Psi_{4}(a-5)+5 \Psi_{4}(a-4)+5 \Psi_{4}(a-3)+\Psi_{4}(a-2) .
$$

$$
2 \mathbf{E}\left\{\omega_{n}(a)\right\}=\mathbf{E}\left\{\left(\begin{array}{c}
\tau_{n}(a-2) \\
2
\end{array}\right)\right\}+\mathbf{E}\left\{\left(\begin{array}{c}
\tau_{n}(a-1) \\
2
\end{array}\right)\right\}
$$

$$
\begin{gathered}
8 \mathbf{E}\left\{\left(\begin{array}{c}
\omega_{n}(a) \\
2
\end{array}\right)\right\}=\mathbf{E}\left\{\left(\begin{array}{c}
\tau_{n}(a-5) \\
4
\end{array}\right)\right\}+5 \mathbf{E}\left\{\left(\begin{array}{c}
\tau_{n}(a-4) \\
4
\end{array}\right)\right\} \\
+5 \mathbf{E}\left\{\left(\begin{array}{c}
\tau_{n}(a-3) \\
4
\end{array}\right)\right\}+\mathbf{E}\left\{\left(\begin{array}{c}
\tau_{n}(a-2) \\
4
\end{array}\right)\right\} .
\end{gathered}
$$

Theorem 2: If $\alpha>0$ and $r \geq 1$, then

$$
\lim _{n \rightarrow \infty} \mathbf{E}\left\{\left(\frac{\omega_{n}([\alpha \sqrt{n}])}{n}\right)^{r}\right\}=\frac{m_{2 r}(\alpha)}{2^{r} r !}
$$

where $m_{r}(\alpha)$ is given by (16).

Proof: Since $g_{j}(1)=C_{j-1} / 2$ for $j=1,2, \ldots$ where $C_{j-1}$ is defined by (36) we have

$$
L_{r}(1)=2^{2 r}-2 \sum_{j=1}^{r} C_{j-1} 2^{2 r-2 j}=\left(\begin{array}{c}
2 r \\
r
\end{array}\right)
$$

if $r \geq 1$. If in (80), $a=[\alpha \sqrt{n}], a>0$, and $r \geq 1$, we obtain that

or

$$
\mathbf{E}\left\{\left(\begin{array}{c}
\omega_{n}(a) \\
r
\end{array}\right)\right\} \sim \mathbf{E}\left\{\left(\begin{array}{c}
\tau_{n}(a) \\
2 r
\end{array}\right)\right\}\left(\begin{array}{c}
2 r \\
r
\end{array}\right) \frac{1}{2^{r}}
$$

$$
\mathbf{E}\left\{\left[\omega_{n}(a)\right]^{r}\right\} \sim \mathbf{E}\left\{\left[\tau_{n}(a)\right]^{2 r}\right\} \frac{1}{2^{r} r !}
$$

as $n \rightarrow \infty$. This proves (85), and (9) follows from (85).

\section{The Sojourn Time $\omega_{n}^{*}(a)$}

We defined $\omega_{n}^{*}(a)$ as the number of subscripts $r=1,2, \ldots, n$ for which $\left|\zeta_{r}\right| \geq a$ where $a=1,2, \ldots$. Let us associate a stochastic process $\{\zeta(t), t \geq 0\}$ with the random walk $\left\{\zeta_{r}, r \geq 0\right\}$. We say that the process $\{\zeta(t), t \geq 0\}$ is in state $B$ in the interval $[r, r+1)$ if $\left|\zeta_{r}\right| \geq a$, and in state $A$ if $\left|\zeta_{r}\right|<a$ where $a=1,2, \ldots$. Then the process $\{\zeta(t), t \geq 0\}$ alternately is in the states $A$ and $B$, and we can interpret $\omega_{n}^{*}(a)$ as $\beta(n+1)$ where $\beta(n+1)$ is the total time that the process $\{\zeta(t), t \geq 0\}$ spends in state 
$B$ in the interval $(0, n+1)$. If $\alpha_{1}, \beta_{1}, \alpha_{2}, \beta_{2}, \ldots$ denote the lengths of the successive intervals spent in states $A$ and $B$ respectively, then $\left\{\alpha_{i}\right\}$ and $\left\{\beta_{i}\right\}$ are independent sequences of independent random variables. Now $\alpha_{1}$ has the same distribution as $\Theta(a, a) ; \alpha_{i},(i \geq 2)$, has the same distribution as $\Theta(1,2 a-1)$, and $\beta_{i},(i \geq 1)$, has the same distribution as $\rho(1)$. The random variables $\Theta(a, b)$ and $\rho(a)$ are defined in Section 2. If we use the notation

$$
R_{a, b}(w)=\mathbf{E}\left\{w^{\Theta(a, b)}\right\}=\frac{[\gamma(w)]^{a}+[\gamma(w)]^{b}}{1+[\gamma(w)]^{a+b}}
$$

where $a \geq 1$ and $b \geq 1$, then by (45) we can write that

$$
(1-w)(1-z w) \sum_{n=0}^{\infty} \mathbf{E}\left\{z^{\omega_{n}^{*}(a)}\right\} w^{n}=1-z w-(1-z) \frac{R_{a, a}(w)[1-\gamma(z w)]}{1-\gamma(z w) R_{1,2 a-1}(w)}
$$

or

$$
(1-w) \sum_{n=0}^{\infty} \mathbf{E}\left\{z^{\omega_{n}^{*}(a)}\right\} w^{n}=1-2[\gamma(w)]^{a}\left(\frac{1-z}{1-z w}\right)\left(\frac{1-\gamma(z w)}{A-B \gamma(z w)}\right)
$$

if $|w|<1$ and $|z w|<1$ where $A=1+[\gamma(w)]^{2 a}$ and $B=\gamma(w)+[\gamma(w)]^{2 a-1}$.

By forming the $r$ th derivative of (91) with respect to $z$ at $z=1$ we obtain that

$$
\Phi_{r}^{*}(a)=(1-w) \sum_{n=0}^{\infty} \mathbf{E}\left\{\left(\begin{array}{c}
\omega_{n}^{*}(a) \\
r
\end{array}\right)\right\} w^{n}=\frac{2^{r} \gamma^{a+r-1}\left(1+\gamma^{2}\right)}{\left(1-\gamma^{2}\right)^{2 r}} U_{r}(\gamma)
$$

for $a \geq 1, r \geq 1$ and $|w|<1$ where

$$
\begin{gathered}
U_{r}(\gamma)=(1+\gamma)^{2 r-1}-\sum_{s=1}^{r-1} \sum_{\nu=1}^{s}\left(1-\gamma^{2 a-1}\right)\left(\gamma+\gamma^{2 a-1}\right)^{\nu-1} \\
\cdot\left(1+\gamma^{2}\right)^{\nu}(1+\gamma)^{2 r-2 s-1} \nu ! Q_{s, \nu}(\gamma) / s !
\end{gathered}
$$

is a polynomial in $\gamma$. In (92) and (93), $\gamma=\gamma(w)$ is defined by (33) and $Q_{s, \nu}(\gamma)$ by (52).

If we use the notation (63), we can write that

$$
2^{r-1} \Phi_{r}^{*}(a)=\Psi_{2 r}(a-3 r+1) U_{r}(\gamma)
$$

If we take into consideration that $\Psi_{r}(a) \gamma=\Psi_{r}(a+1)$ for $a \geq 1$ and $r \geq 1$, then the right-hand side of (94) can be expressed as a linear combination of $\Psi_{2 r}((2 j-1) a-$ $3 r+k$ ) for $j=1,2, \ldots, r$ and $k=1,2, \ldots, 3 r$. By forming the coefficient of $w^{n}$ on both sides of (94) we can express the $r$ th binomial moment of $\omega_{n}^{*}(a)$ as a linear combination of the $2 r$ th binomial moments of $\tau_{n}((2 j-1) a-3 r+k)$ for $j=1,2, \ldots, r$ and $k=1,2, \ldots, 3 r$.

Theorem 3: If $\alpha>0$ and $r \geq 1$, then

$$
\lim _{n \rightarrow \infty} \mathbf{E}\left\{\left(\frac{\omega_{n}^{*}([\alpha \sqrt{n}])}{n}\right)^{r}\right\}=M_{r}^{*}(\alpha)
$$

exists and $M_{r}^{*}(\alpha)$ is given by $(10)$. 
Proof: By (39) and (54) we can prove that

$$
\begin{gathered}
2^{r} x\left\{1-\sum_{s=1}^{r-1} \quad \sum_{\nu=1}^{s}\left(1-x^{2}\right)\left(1+x^{2}\right)^{\nu-1} 2^{\nu} \nu ! Q_{s, \nu}(1) /\left(2^{2 s} s !\right)\right\} \\
=\frac{4}{2^{r}} \sum_{j=1}^{r}\left(\begin{array}{c}
2 r-1 \\
r-j
\end{array}\right) x^{2 j-1}
\end{gathered}
$$

for $r \geq 1$ and therefore if in (94) we put $a=[\alpha \sqrt{n}], \alpha>0$, we obtain that

$$
\mathbf{E}\left\{\left(\begin{array}{c}
\omega_{n}^{*}(a) \\
r
\end{array}\right)\right\} \sim \frac{4}{2^{r}} \sum_{j=1}^{r}\left(\begin{array}{c}
2 r-1 \\
r-j
\end{array}\right) \mathbf{E}\left\{\left(\begin{array}{c}
\tau_{n}((2 j-1) a) \\
2 r
\end{array}\right)\right\}
$$

as $n \rightarrow \infty$ or

$$
\mathbf{E}\left\{\left[\omega_{n}^{*}(a)\right]^{r}\right\} \sim \frac{(r-1) !}{2^{r-1}} \sum_{j=1}^{r} \frac{1}{(r-j) !(r+j-1) !} \mathbf{E}\left\{\left[\tau_{n}((2 j-1) a)\right]^{2 r}\right\}
$$

as $n \rightarrow \infty$. We obtain (10) from (98) by making use of (24) and (26).

Clearly, (23) and (95) imply (8).

Theorem 4: If $\alpha>0$, then

$$
\lim _{n \rightarrow \infty} \mathbf{P}\left\{\frac{\omega_{n}^{*}([\alpha \sqrt{n}])}{n} \leq x\right\}=G_{\alpha}(x)
$$

for $0<x<1$ where $G_{\alpha}(x)$ is given by (11) for $0 \leq x<1$ and $G_{\alpha}(1)=1$.

Proof: By (9) and (10) we can write that

$$
M_{r}^{*}(\alpha)=2 r !(r-1) ! \sum_{k=1}^{r} \frac{1}{(r-k) !(r+k-1) !} M_{r}((2 k-1) \alpha)
$$

for $r \geq 1$ and $\alpha>0$, and

$$
M_{r}(\alpha)=r \int_{0}^{1} x^{r-1}\left[1-F_{\alpha}(x)\right] d x
$$

for $r \geq 1$ and $\alpha>0$ where the distribution function $F_{\alpha}(x)$ is given by (12). We shall determine $G_{\alpha}(x)$ by using Laplace-Stieltjes transforms.

Let us define

$$
\Psi_{\alpha}^{*}(s)=\int_{-0}^{1} e^{-s x} d G_{\alpha}(x)
$$

for $\operatorname{Re}(s) \geq 0$. Since

$$
\Psi_{\alpha}^{*}(s)=1+\sum_{r=1}^{\infty} \frac{(-1)^{r} s^{r}}{r !} M_{r}^{*}(\alpha)
$$

for $\operatorname{Re}(s) \geq 0$, by (100), (101), (55) and (56) we obtain that

$$
\Psi_{\alpha}^{*}(s)=1+2 \sum_{r=1}^{\infty} \sum_{k=1}^{r} \frac{(-1)^{r} s^{r} r !}{(r-k) !(r+k-1) !} \int_{0}^{1} x^{r-1}\left[1-F_{(2 k-1) \alpha}(x)\right] d x
$$




$$
\begin{gathered}
=1+2 \sum_{k=1}^{\infty} \sum_{r=k}^{\infty} \frac{(-1)^{r} s^{r} r !}{(r-k) !(r+k-1) !} \int_{0}^{1} x^{r-1}\left[1-F_{(2 k-1) \alpha}(x)\right] d x \\
=1+2 \sum_{k=1}^{\infty}(-1)^{k} s^{k} \int_{0}^{1}\left(\sum_{j=0}^{\infty} \frac{(-1)^{j} s^{j}(j+k) ! x^{j+k-1}}{j !(j+2 k-1) !}\right)\left[1-F_{(2 k-1) \alpha}(x)\right] d x \\
=1+2 \sum_{k=1}^{\infty} \frac{(-1)^{k} s^{k} k !}{(2 k-1) !} \int_{0}^{1} e^{-s x} x^{k-1} M(k-1,2 k, s x)\left[1-F_{(2 k-1) \alpha}(x)\right] d x .
\end{gathered}
$$

Now by (55),

$$
M(k-1,2 k, s x)=\sum_{j=0}^{\infty} Q_{k}(j)(s x)^{j}
$$

where $Q_{k}(0)=1, Q_{k}(1)=(k-1) /(2 k)$ if $k \geq 1, Q_{1}(j)=0$ if $j \geq 1$, and

$$
Q_{k}(j)=\frac{(2 k-1) !(k+j-2) !}{(k-2) !(2 k+j-1) ! j !}
$$

for $k \geq 2$ and $j \geq 1$. By using (105) we can write that

$$
\begin{aligned}
& \Psi_{\alpha}^{*}(s) \\
& =1+2 \sum_{k=1}^{\infty} \frac{(-1)^{k} k !}{(2 k-1) !} \sum_{j=0}^{\infty} Q_{k}(j) s^{k+j} \int_{0}^{1} e^{-s x} x^{k+j-1}\left[1-F_{(2 k-1) \alpha}(x)\right] d x \\
& =1+2 \sum_{k=1}^{\infty} \frac{(-1)^{k} k !}{(2 k-1) !} \sum_{j=0}^{\infty}(-1)^{k+j} Q_{k}(j) \int_{0}^{1}\left(\frac{d^{k+j} e^{-s x}}{d x^{k+j}}\right) \\
& x^{k+j-1}\left[1-F_{(2 k-1) \alpha}(x)\right] d x \\
& =1+2 \sum_{k=1}^{\infty} \frac{(-1)^{k} k !}{(2 k-1) !} \sum_{\ell=k}^{\infty}(-1)^{\ell} Q_{k}(\ell-k) \int_{0}^{1}\left(\frac{d^{\ell} e^{-s x}}{d x^{\ell}}\right) \\
& \cdot x^{\ell-1}\left[1-F_{(2 k-1) \alpha}(x)\right] d x \\
& =1+4 \sum_{k=1}^{\infty} \frac{(-1)^{k} k !}{(2 k-1) !} \sum_{\ell=k}^{\infty} Q_{k}(\ell-k)(\ell-1) ![1-\Phi((2 k-1) \alpha)] \\
& +2 \sum_{k=1}^{\infty} \frac{(-1)^{k} k !}{(2 k-1) !} \sum_{\ell=k}^{\infty} Q_{k}(\ell-k) \int_{0}^{1} e^{-s x} \frac{d^{\ell} x^{\ell-1}\left[1-F_{(2 k-1) \alpha}(x)\right]}{d x^{\ell}} d x .
\end{aligned}
$$

By (107) we can conclude that

$$
G_{\alpha}(0)=1+4 \sum_{k=1}^{\infty} \frac{(-1)^{k} k !}{(2 k-1) !}\left(\sum_{\ell=k}^{\infty} Q_{k}(\ell-k)(\ell-1) !\right)[1-\Phi((2 k-1) \alpha)]
$$


for $\alpha>0$. By using (57) we can prove that (108) is indeed equal to (14). Furthermore, we have

$$
\frac{d G_{\alpha}(x)}{d x}=2 \sum_{k=1}^{\infty} \frac{(-1)^{k} k !}{(2 k-1) !} \sum_{\ell=k}^{\infty} Q_{k}(\ell-k) \frac{d^{\ell} x^{\ell-1}\left[1-F_{(2 k-1) \alpha}(x)\right]}{d x^{\ell}}
$$

if $0<x<1$.

By (107) it follows also that

$$
G_{\alpha}(x)=1+2 \sum_{k=1}^{\infty} \frac{(-1)^{k} k !}{(2 k-1) !} \sum_{\ell=k}^{\infty} Q_{k}(\ell-k) \frac{d^{\ell-1} x^{\ell-1}\left[1-F_{(2 k-1) \alpha}(x)\right]}{d x^{\ell-1}}
$$

for $0 \leq x<1$ and $\alpha>0$. This proves (99).

Finally, by (23) and (99) we can conclude that $\mathbf{P}\left\{\omega^{*}(\alpha) \leq x\right\}=G_{\alpha}(x)$ and $G_{\alpha}(x)$ is given by (11) for $\alpha>0$ and $0 \leq x<1$.

\section{The Brownian Meander}

The distribution of the sojourn time for the Brownian meander can be obtained in the same way as we found the distribution of $\omega^{*}(\alpha)$ for the Brownian motion. Let $\left\{\xi^{+}(t), 0 \leq t \leq 1\right\}$ be a standard Brownian meander such that $\mathbf{P}\left\{\xi^{+}(0)=0\right\}=1$ and $\mathbf{P}\left\{\xi^{+}(t) \geq 0\right\}=1$ for all $0 \leq t \leq 1$. Define

$$
\omega^{+}(\alpha)=\int_{0}^{1} \delta\left(\xi^{+}(t)>\alpha\right) d t
$$

for $\alpha \geq 0$. We can prove that

$$
\begin{aligned}
& \mathbf{E}\left\{\left[\omega^{+}(\alpha)\right]^{r}\right\}=\frac{4 r !}{2^{r}} \sqrt{\frac{\pi}{2}} \sum_{j=1}^{r} \frac{(-1)^{j-1}}{(r-j) !(r+j) !} \\
& \cdot\left[(r+j) m_{2 r-1}((2 j-1) \alpha)-j m_{2 r-1}(2 j \alpha)\right]
\end{aligned}
$$

for $r \geq 1$ and $\alpha>0$ where $m_{r}(\alpha)$ is given by (16).

The moments (112) uniquely determine the distribution of $\omega^{+}(\alpha)$ and we have

$$
\begin{gathered}
\mathbf{P}\left\{\omega^{+}(\alpha) \leq x\right\}=2 \sum_{\ell=1}^{\infty} \sum_{k=1}^{\ell} \frac{k(k+1) !}{(2 k) !} A_{k}(\ell-k) \frac{d^{\ell-1} x^{\ell-1}\left[1-F_{k \alpha}(x)\right]}{d x^{\ell-1}} \\
-4 \sum_{\ell=1}^{\infty} \sum_{k=1}^{\ell} \frac{k(k+1) !}{(2 k) !} B_{k}(\ell-k) \frac{d^{\ell-1} x^{\ell-1}\left[1-F_{(2 k-1) \alpha / 2}(x)\right]}{d x^{\ell-1}}
\end{gathered}
$$

for $0 \leq x<1$, and $\mathbf{P}\left\{\omega^{+}(\alpha) \leq 1\right\}=1$ where $F_{\alpha}(x)$ is defined by (12) and (13). The coefficients $A_{k}(j)$ and $B_{k}(j)$ are defined by the series

$$
M(k-1,2 k+1, x)=\sum_{j=0}^{\infty} A_{k}(j) x^{j}
$$


and

and determined by (55).

$$
M(k-2,2 k, x)=\sum_{j=0}^{\infty} B_{k}(j) x^{j}
$$

\section{References}

[1] Abramowitz, M. and Stegan, I.A., Handbook of Mathematical Functions, Dover, New York 1970.

[2] Donsker, M.D., An invariance principle for certain probability limit theorems, Four Papers on Probability. Memoirs of the AMS 6 (1951), 1-12.

[3] di Bruno, Faá, Note sur une nouvelle formule du calcul differential, Quart. J. of Math. 1 (1855), 359-360.

[4] Gikhman, I.I. and Skorokhod, A.V., Introduction to the Theory of Random Processes, W.B. Saunders, Philadelphia 1969.

[5] Jordan, Ch., Calculus of Finite Differences, Budapest 1939. [Reprinted by Chelsea, New York 1947.]

[6] Kac, M., On distributions of certain Wiener functionals, Trans. of the AMS 65 (1949), 1-13.

[7] Knight, F.B., Random walk and a sojourn density process of Brownian motion, Trans. of the AMS 109 (1965), 56-86.

[8] Laplace, P.S., Théorie Analytique des Probabilités. Courcier, Paris 1812. [Reprinted by Culture et Civilisation, Bruxelles 1962.]

[9] Lévy, P., Sur certains processus stochastiques homogènes, Compositio Mathematica 7 (1939), 283-339.

[10] Slater, L.J., Confluent Hypergeometric Functions, Cambridge University Press 1960.

[11] Takács, L., On the classical ruin problems, J. of the American Statistical Assoc. 64 (1969), 889-906.

[12] Takács, L., Fluctuation problems for Bernoulli trials, SIAM Review 21 (1979), 222-228.

[13] Takács, L., On the local time of the Brownian motion, The Annals of Applied Probability 5 (1995), 741-756.

[14] Takács, L., On a generalization of the arc-sine law, The Annals of Applied Probability 6 (1996), 1035-1040.

[15] Todhunter, I., A History of the Mathematical Theory of Probability from the Time of Pascal to that of Laplace, Cambridge University Press 1865. [Reprinted by Chelsea, New York 1949.] 


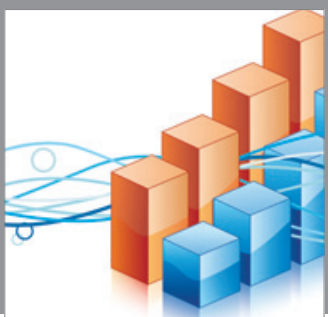

Advances in

Operations Research

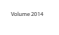

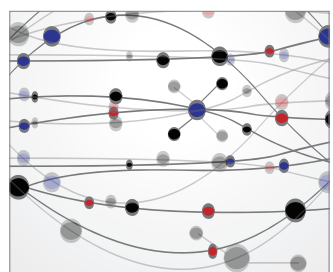

\section{The Scientific} World Journal
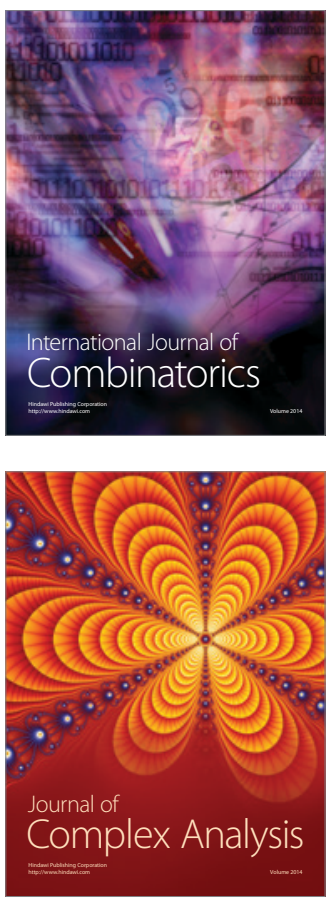

International Journal of

Mathematics and

Mathematical

Sciences
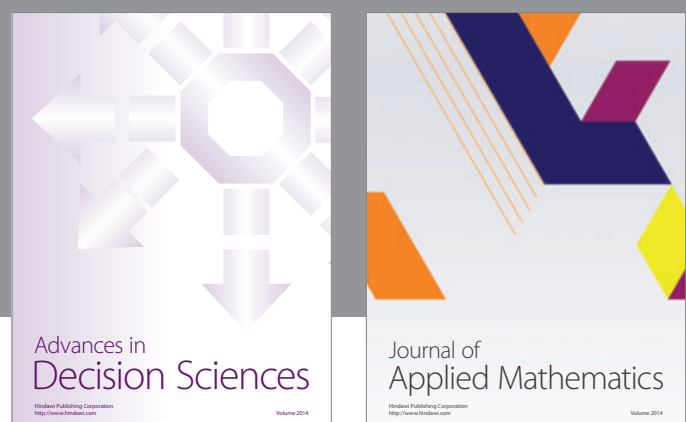

Journal of

Applied Mathematics
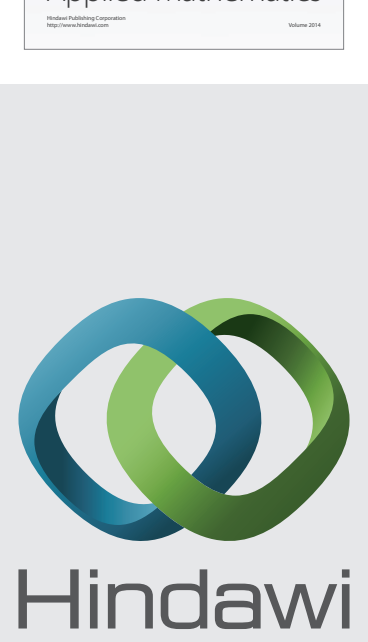

Submit your manuscripts at http://www.hindawi.com
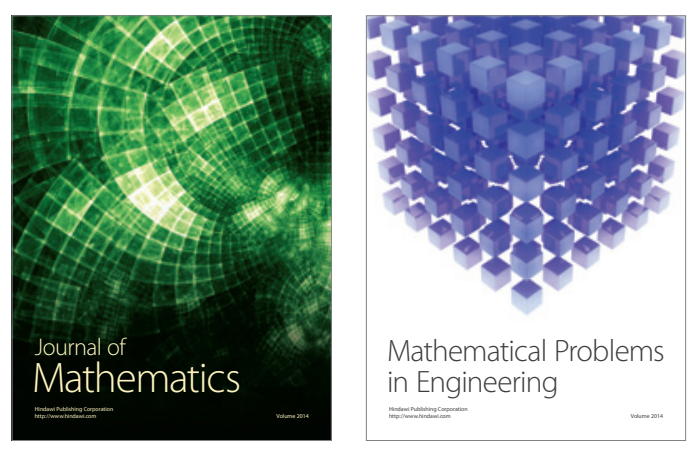

Mathematical Problems in Engineering
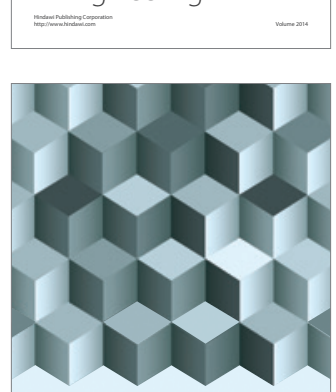

Journal of

Function Spaces
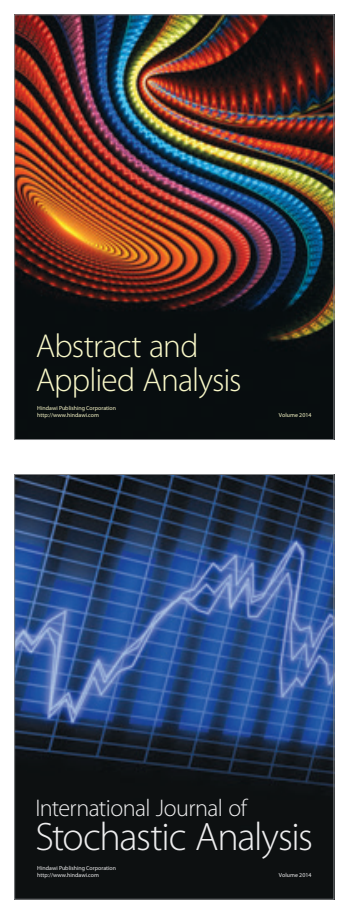

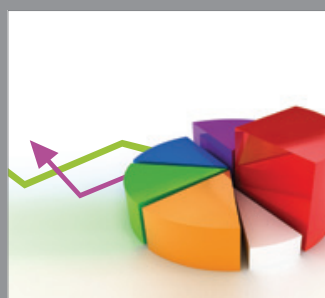

ournal of

Probability and Statistics

Promensencen
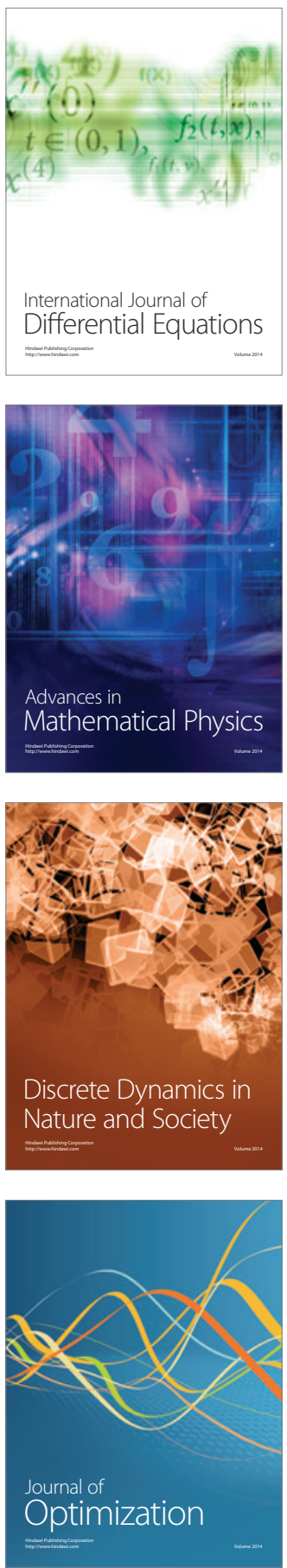